\title{
Analysis of Human Cardiovascular System using Equivalent Electronic System
}

\author{
N. Vinoth ${ }^{1}$, S. Nagarjuna Chary ${ }^{2}$ \\ Dept of Electronics and Instrumentation Engineering, Annamalai University, Annamalai nagar, Tamil Nadu, India ${ }^{1}$ \\ Dept of Electronics and Instrumentation Engg, VNR Vignana Jyothi Institute of Engineering and Technology,
}

Hyderabad, India ${ }^{2}$

\begin{abstract}
This paper describes simulation and analysis of the human cardiovascular system using a complex electronic circuit. In this study we have taken a slightly different approach to the modelling of the system and tried to advance existing electrical models by increasing more segments and parameters. Anatomical and physiological data for circuit parameters have been extracted from medical articles and textbooks. The frequency of heart is $1 \mathrm{~Hz}$ and the system operates in steady state condition. Each artery is modelled by one capacitor, resistor and inductor. The left and right ventricles are modelled using AC power suppliers and diodes. The results of the simulation including pressure graphs exhibit operation of the cardiovascular system under normal condition. From the electrical cardiovascular model we have analysed some cases which reflect the real time functioning of the human cardiovascular system. Hence we have changed the circuit parameters and observed the output responses which satisfy the actual human responses in different situations.
\end{abstract}

Keywords: Model; Simulation; Electronic Circuit; Cardiovascular System; Case Studies.

\section{INTRODUCTION}

The human cardiovascular system is primarily a transport system in which oxygen, carbon dioxide and nutrients are carried by the blood to and from the various muscles and organs. The cardiovascular system consists of two parts they are systemic circuit and the pulmonary circuit which are connected via the heart [1].

Diseases of the human cardiovascular system are one of the main problems in contemporary health care in industrial countries. They cause the majority of deaths and also often afflict people in their most productive age. In this context different approaches were used with the aim of providing better understanding and simulation of the blood flow in the human cardiovascular system [2].

A three parametric model of heart muscle mechanics was introduced [3]. The study of the series elasticity of cardiac muscle was presented. Afterwards, the time-varying elastance model of the left ventricle was introduced and later the relationship between pressure-volume area and cardiac oxygen consumption was described. Finite element methods are commonly used to simulate the left ventricular performance [4].

The mathematical description of the whole human cardiovascular system remains a complex task and for that reason models are simplified with respect to particular parts of interest are presented [5]. The model which is presented here describes simulation of the cardiovascular system using a complex electronic circuit [6].
In this study, we have taken a slightly different approach to the modelling of the system. We have tried to advance existing electrical models by increasing more segments and parameters.

Finally we have analysed an included some case studies on these model, by changing the circuit parameters and the AC sources (pace makers) and observed the output responses which obey the actual human responses in various cases. Therefore, normal and abnormal performance of arterial system can be studied.

\section{MODEL SIMULATION PRINCIPLES}

In our model, every blood, vessel, atrium, ventricle and set of all capillaries and arterioles have been presented by a block consisting of a resistor, an inducer and a capacitor. Voltage, current, charge, resistance and capacitance in the electronic circuit are equivalent to blood pressure, flow, volume, resistance and compliance in the cardiovascular system [7].

Ground potential (reference for voltage measurements) is equivalent to zero. The correlation between electrical characteristics of the system and their mechanical counterparts are as follow:

$$
\begin{array}{ll}
1 \mathrm{mmHg} & =1 \text { volt }(\text { pressure } \sim \text { voltage }) \\
1 \mathrm{ml} & =10 \mu \mathrm{As}(\text { volume } \sim \text { charge }) \\
0.01 \mathrm{ml} / \mathrm{Pa} & =1 \mu \mathrm{F}(\text { compliance } \sim \text { capacitance })
\end{array}
$$




\section{IJIREEICE \\ International Journal of Innovative Research in Electrical, Electronics, Instrumentation and Control Engineering \\ ISO 3297:2007 Certified \\ Vol. 5, Issue 2, February 2017}

$$
\begin{array}{ll}
1 \mathrm{~Pa} . \mathrm{s} / \mathrm{ml} & =1 \mathrm{k} \Omega(\text { resistance }) \\
1 \mathrm{~Pa} . \mathrm{s} 2 / \mathrm{ml} & =1 \mu \mathrm{H}(\text { inertia } \sim \text { inducer }) \\
1 \mathrm{~s} & =1 \mathrm{~s}(\text { unit of time) }
\end{array}
$$

In cardiovascular and arterial system, blood vessels resistance depending on blood viscosity and vessel diameter is simulated by resistors. The vessel compliance is simulated by capacitors. The blood inertia is considered using inducers.

Atriums are simulated as part of the venous system without any contraction. Atriums are modelled as a resistor-capacitor segment. Ventricles are simulated as a section of blood vessels in which its resting capacitance (diastole) can be decreased (systole) and then returned to the resting capacitance [8].

Essentially, energy of systolic contraction of left and right ventricles is modelled by superposition of three AC power supplies and diodes. These voltage sources are amplifiers and the inputs of them are connected to the capacitors simulating atriums.

Thus, during systole, the voltage from the ventricular capacitor is amplified and applied to the aortic capacitor. As can be seen, each block consists of a resistor (R), an inducer (L) and a capacitor (C).

\section{SYSTEM DESCRIPTION}

The equivalent circuit of the cardiovascular system [1] is shown in Fig.1. In this simulation, blood volume, cardiac output and aortic pressure should be $5000 \mathrm{ml}, 83 \mathrm{ml} / \mathrm{s}$ and 80-120 mmHg. To facilitate the understanding of the circuit, the anatomic structures are brought into it.

Similarly as the preparation in vivo, also the equivalent circuit can be subdivided into two parts: heart (atrium and ventricle) and the arterial circulation. The left atrium and ventricle are represented by two capacitors $101 \mu \mathrm{F}$ and 25 $\mu \mathrm{F}$. Also right atrium and ventricle are modelled by two capacitors $216.45 \mu \mathrm{F}$ and $150 \mu \mathrm{F}$.

Using of suitable diodes were very important matter in this circuit. The aortic, mitral, tricuspid and pulmonary valve are simulated with ideal diodes. Using these types of diodes has helped the circuit to show the operation of the cardiovascular system properly. The contracting relaxing actions of the left and right ventricles are achieved by the pacemaker.

The pacemaker consists of three AC power supply and two diodes (DIN4148).The pacemaker has the pulse generator of $1 \mathrm{~Hz}$. For each ventricle, one pace maker is used in order to drive the voltage into the circuit. As it can be seen in the Fig. 2, an 8V and 7V DC voltage source is used for the right and left ventricle to adjust variation of pressure between $8-25$ and $7-120$ volt $(\mathrm{mmHg})$ for it.
The values of $\mathrm{R}, \mathrm{L}$ and $\mathrm{C}$ were either directly extracted from [7 -9] The circuit consists of the biggest arteries and arterioles have been simulated as one separated compartment with resistance of $72 \mathrm{k} \Omega$ and capacitance of $1.4 \mu \mathrm{F}$. The capillaries and veins are also modeled by one and two segments.

The upper body arteries such as carotid have been attached to arterioles by one connection. The generated current of suppliers distributes to left ventricle, aorta and upper body arteries. It continues its path toward the body arteries such as femoral, renal or gastric [9].

The current passes the arterioles, capillaries and veins and enters to right atrium. Another amplifier, pacemaker, generates the required current for circulation in the pulmonary arteries and veins and the final current enters the left atrium finally. It shall be noted that there is no leakage of charge in the system and the output voltage is proportional to the input voltage.

The proportionality factor reflects the contractility of the ventricles. To meet this end both terminals of ventricles capacitors are connected to the pace makers connections. Due to their high input impedance, there is no appreciable leakage of charge from the circuit. In this circuit there is no point of constant pressure (voltage). Therefore, total capacitance of the system $(905 \mu \mathrm{F})$ is one of the important determinants of its functions.

\section{RESULTS AND DISCUSSION}

From the electrical cardiovascular model we have analyzed some cases which reflect the real time functioning of the human cardiovascular system. The model is capable of showing pressure and volume signals for different arteries throughout carotid to femoral triggered by initial systolic contraction.

Fig. 2 shows the pressure (voltage) graphs and it can be obtained from the different points of the circuit. Hence we have changed the circuit parameters and observed the output responses which satisfy the actual human responses in different situations. Here we have included some case studies they are as follows.

Case 1: First we have taken the response for a normal person. The responses are in good agreement with the standard human pressure waveforms.

The figure 3 responses show the pressure distribution of the 1. Aorta. 2. Left Ventricle \& 3. Right Ventricle with average pressures of 120/80, 120/8 \& 25/8 respectively this is for normal person without any abnormalities.

Case 2: The person effecting with myocardial infarction. This is generally due to the hypertension or sudden raise in the BP (Blood Pressure). 
International Journal of Innovative Research in Electrical, Electronics, Instrumentation and Control Engineering

ISO 3297:2007 Certified

Vol. 5, Issue 2, February 2017

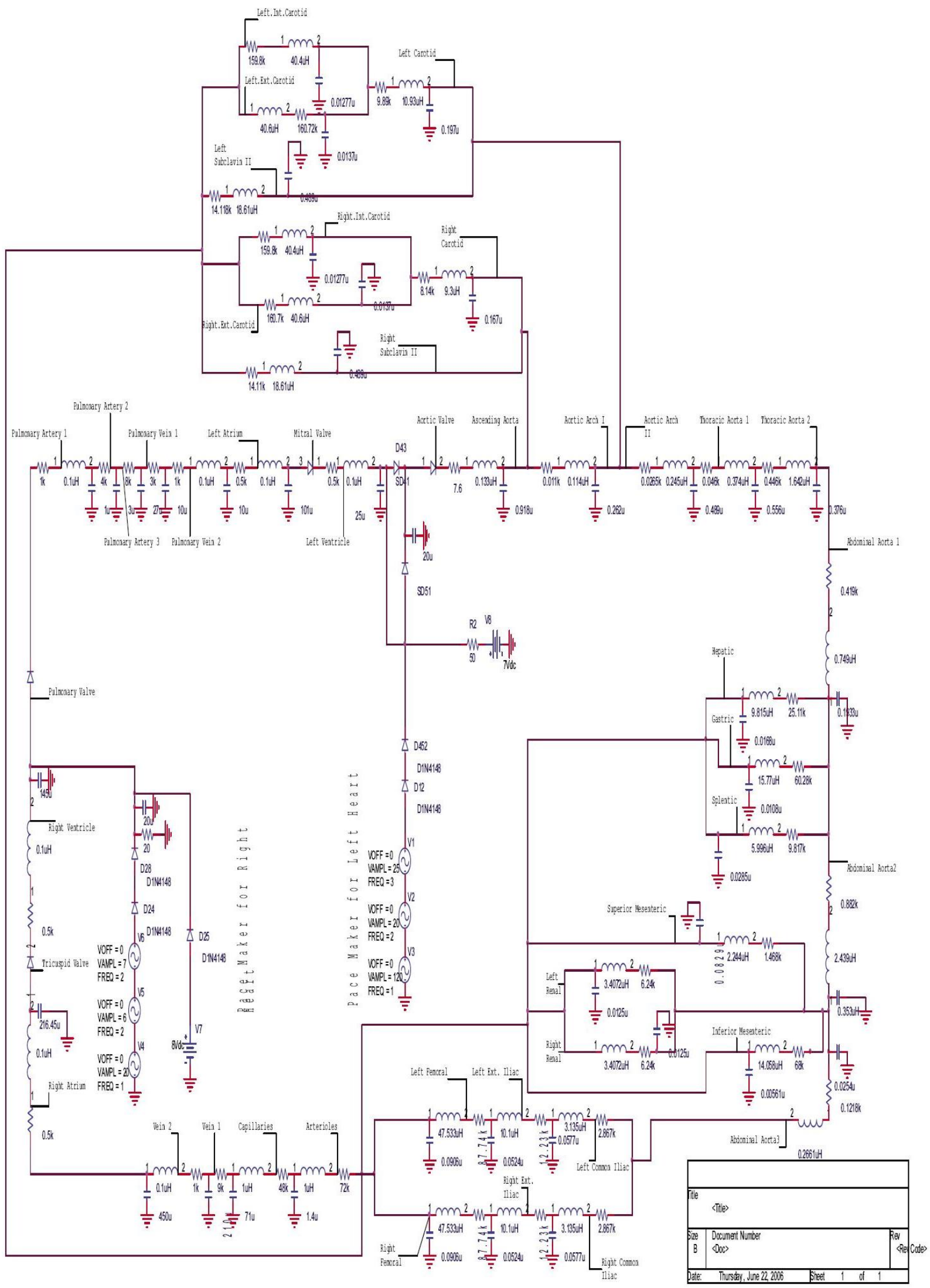

Fig.1 Electronic circuit of cardiovascular system 


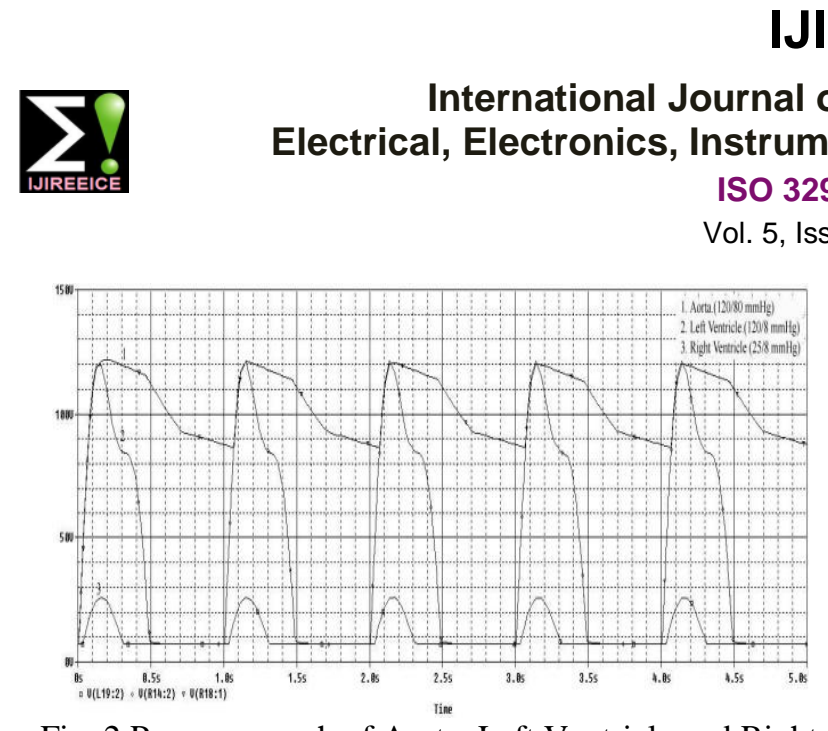

Fig. 2 Pressure graph of Aorta, Left Ventricle and Right Ventricle for frequency of $1 \mathrm{~Hz}$

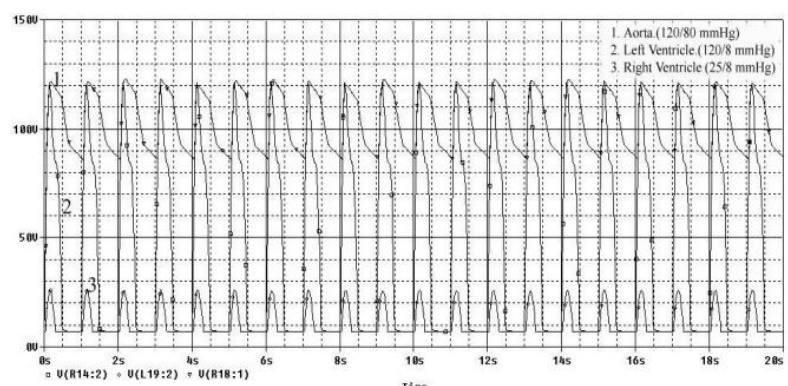

Fig.3 Pressure waveform for a normal person

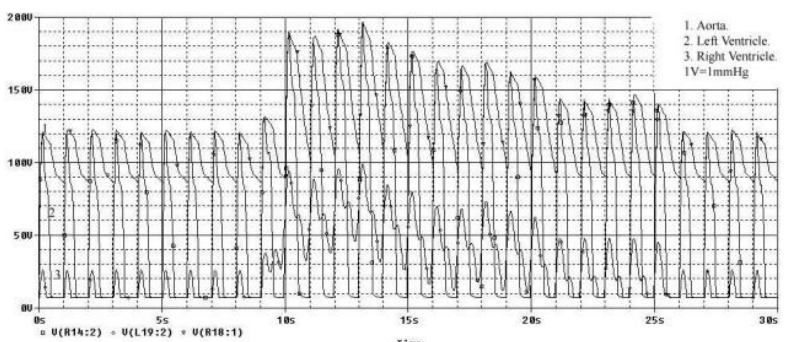

Fig.4 Pressure waveform for a myocardial infarction person

The above responses show the pressure distribution of the 1. Aorta. 2. Left Ventricle \& 3. Right Ventricle with pressures varies from $120 / 80$ to $190 / 100,120 / 8$ to $120 / 8$ \& $25 / 8$ to $100 / 50$ respectively. This occurs when a person effects with sudden stroke in the heart or myocardial infarction.

Case 3: The person effecting with myocardial infarction and then after making necessary treatment. This is generally when the person get treatment after myocardial infarction.



Fig. 5 Pressure waveform for a myocardial infarction person after medication
The above responses show the pressure distribution of the 1. Left ventricle $\& 2$. Right Ventricle with pressures varies from 120/80, 120/8 \& 25/8 respectively. This occurs when a person effects with sudden stroke and then gets proper treatment in the heart.

Case 4: The person effecting with high blood pressure. This is generally when the person gets hypertension or by the genetic disease.



Fig. 6 Pressure waveform for high blood pressure person

The above responses show the pressure distribution of the 1. Aorta. 2. Left Ventricle \& 3. Right Ventricle with pressures varies from 175/100, 175/8 \& 50/8 respectively. This occurs when a person effects with high blood pressure or from the genetic disease. Generally this occurs for the people averagely above the age of 35 .

Case 5: The person effecting with low blood pressure. This is generally when the person gets weak or by the genetic disease.

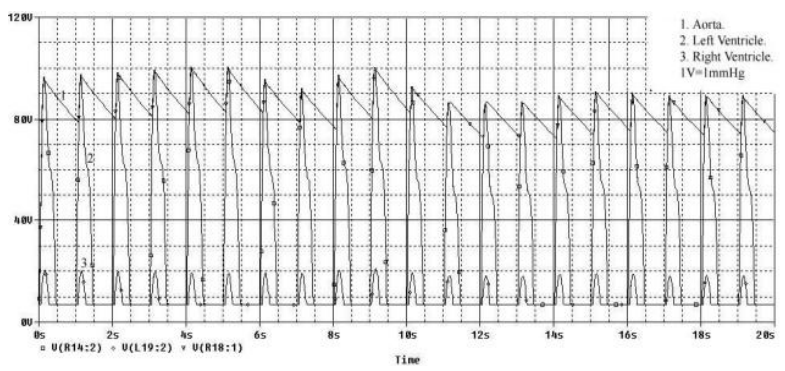

Fig. 7 Pressure waveform for low blood pressure person

The above responses show the pressure distribution of the 1. Aorta. 2. Left Ventricle \& 3. Right Ventricle with pressures varies from 95/80, 95/8 \& 20/8 respectively. This occurs when a person effects with low blood pressure or from the genetic disease. Generally this occurs for the people who are very weak.

\section{CONCLUSION}

In this paper simulation and analysis of the human cardiovascular system using a complex electronic circuit is done. Modelling of the system is done and tried increasing more segments and parameters.

From the electrical cardiovascular model we have analysed five cases which reflect the real time functioning of the human cardiovascular system. We have changed the circuit parameters, inputs and observed the output responses which satisfy the actual human responses in 


\section{IJIREEICE \\ International Journal of Innovative Research in Electrical, Electronics, Instrumentation and Control Engineering \\ ISO 3297:2007 Certified \\ Vol. 5, Issue 2, February 2017}

different situations. In future work we are going to introduce action potential as source in the place of $1 \mathrm{HZ}$ AC source to drive the circuit and analyse the circuit.

\section{REFERENCES}

[1]. Kamran Hassani, Mahdi Navidbakhsh, Mostafa. Rostami, "Simulation of the cardiovascular system using equivalent Electronic system", Biomed Pap Med Fac Univ Palacky Olomouc Czech Repub, vol.150 (1), pp105-112,2006.

[2]. Zacek M, and Krause E, "Numerical simulation of the blood flow in the human cardiovascular system", Journal of biomechanics, vol.32, pp13-20, 1996.

[3]. Rideout VC, Mathematical and computer modeling of physiological system,. New York: Prentice Hall, 1991.

[4]. Rupnic M, and Runvove F, "Simulation of steady state and transient phenomena by using the equivalent electronic circuit", journal of Computer methods and programs in biomedici vol.67,pp $1-12,2002$.

[5]. T.Helde,t and E.Shim, "Modeling of cardiovascular response to orthostatic stress" Journal of applied physiology, vol. 92, pp 123954,2002 .

[6]. Nebot A, Cellier FE, and Vallverdu M, "Mixed quantitive/qualitive modeling of the cardiovascular system and simulation of it", Journal of computer methods and programs in biomedicine vol.55, pp127-55, 1988.

[7]. Mukkamala R, and Cotton RJ, "A forward model-based validation of cardiovascular system identification", Journal of phisiol heart circ phisiol, vol. 281, pp.2714-30,2001

[8]. Nardinochini P, Pontrelli G, and Teresi L, "A one-dimensional model for blood flow in pre stressed vessel", European Journal of Mechanics vol.24, pp 23-33, 2005.

[9]. Torii R, Oshima M, and Kobayashi T, "Computer modeling of cardiovascular fluid-structure interactions with the deforming spatial-domain-time formulation", Computer methods in Applied Mechanics and Engineering vol.13, pp 549-57. 